\title{
A Personalized 12-week "Brain Fitness Program" for Improving Cognitive Function and Increasing the Volume of Hippocampus in Elderly with Mild Cognitive Impairment
}

\author{
M. Fotuhi ${ }^{1,2}$, B. Lubinski $i^{1,3}$, M. Trullinger ${ }^{4}$, N. Hausterman ${ }^{1,5}$, T. Riloff', M. Hadadi ${ }^{1}$, C.A. Raji

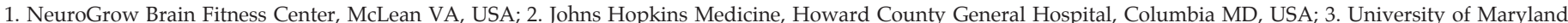

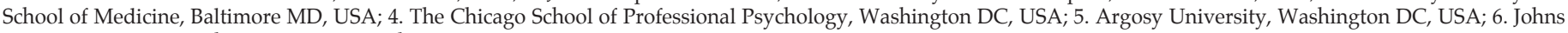
Hopkins Bloomberg School of Public Health, Baltimore MD, USA; 7. University of California Los Angeles, Los Angeles CA, USA

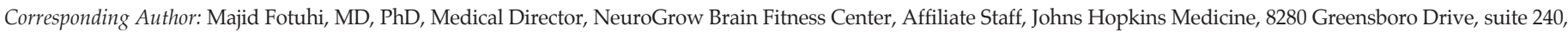
McLean, VA 22102, Phone: 703.462.9296, Fax: 703.462.9269, E-mail: mfotuhi@jhu.edu

J Prev Alz Dis 2016;3(3):133-137

Published online February 8, 2016, http:/ / dx.doi.org/10.14283/jpad.2016.92

\begin{abstract}
Reducing cognitive decline in patients with Mild Cognitive Impairment (MCI) may slow their progression to develop dementia. In this 12-week single-arm intervention trial, elderly patients $(\mathrm{n}=127$, age $70.69+/-10.53,63 \%$ female) with a diagnosis of MCI were enrolled in a multi-disciplinary Brain Fitness Program. The main outcome measure was changes in a battery of 10 cognitive domains. Each patient received weekly personalized cognitive stimulation, neurofeedback training, and brain coaching/counseling for eating a Mediterranean diet, taking omega-3 supplements, increasing fitness, and practicing mindfulness meditation. The post-program testing showed $84 \%$ of the patients experienced statistically significant improvements in their cognitive function $(\mathrm{p}<0.05)$. Among the random sample of 17 patients who had a post-program quantitative MRI, 12 patients had either no atrophy or an actual growth above the baseline volume of their hippocampus. These preliminary findings support the concept that a personalized Brain Fitness Program can improve cognitive function and either reverse or grow the volume of hippocampus in elderly with MCI.
\end{abstract}

Key words: Mild cognitive impairment, rehabilitation, neurofeedback.

\section{Introduction}

$\mathrm{D}$ epression, anxiety, stress, sleep apnea, insomnia, obesity, diabetes, sedentary lifestyle, and low levels of cardiovascular fitness, either alone or in combination, make significant contributions to hippocampal atrophy and cognitive impairment with aging $(1,2)$. Recent clinical trials suggest that some of the atrophy in the hippocampus and the cognitive decline associated with aging or Mild Cognitive Impairment (MCI) can be abated with interventions that increase physical fitness, stimulate cognitive skills, reduce stress, enhance levels of omega-3 fatty acids in the diet, and/or optimize the pattern of brain wave activity (3). These studies suggest that elderly individuals with cognitive impairment benefit significantly from exercise, computer-based cognitive training, and treatment of medical conditions such as sleep apnea, depression, and vitamin deficiencies (low vitamin B12, low vitamin D, and low DHA level), especially if these interventions are combined (4).

Based on the evidence for the multifactorial nature of late-life Alzheimer's disease, and the reported benefits of an integrated approach of targeting all of the causes of cognitive deficiencies in an elderly patient, we set out to determine the benefits of a personalized multidisciplinary "Brain Fitness Program." We provided our patients with MCI with cognitive skills training, counseling, meditation training, and treatment for medical conditions, such as sleep apnea and depression. In this 12-week program, they received weekly neurofeedback treatment, cognitive stimulation, and brain coaching/counseling for stress reduction, diet, exercise, and goal-oriented behavioral modifications. Our rationale was that combining various interventions in an intensive program would result in significant improvements in a short amount of time.

\section{Methods}

\section{Program Description}

An IRB approval was obtained to study the potential benefits of a multi-disciplinary Brain Fitness Program in elderly patients who presented to a community neurology practice in Maryland (USA) and were found to have MCI. The diagnosis of MCI was based on a) a self-reported subjective decline in cognitive function, corroborated by a family member, b) an objective deficit compared to others with the same age and education (by at least 1.5 standard deviation), in one or more formal cognitive tests for memory, executive function, attention, language, or visuospatial skills, and c) preservation of function for activities of daily living and instrumental activities of daily living, and d) no significant impairment in social or occupational activities (i.e. no dementia) 
(5). Those who had mild subjective memory loss and normal cognitive test results, those with moderate to severe Alzheimer's disease or other forms of dementia, and those with significant active comorbid psychiatric conditions such as schizophrenia or bipolar disorder were excluded. Over an 18-month period, 127 patients were enrolled in this trial and signed the consent form. They had an average age $70.69(\mathrm{SD}=10.53), 63 \%$ were women, and had an average baseline Mini-Mental Status Examination (MMSE) score of $27.16(\mathrm{SD}=2.84)$. They did not receive any compensation for participating in this trial.

The Brain Fitness Program consisted of a thorough baseline evaluation followed by three sets of interventions, as described below. In order to monitor patients closely, each patient's progress was documented through a set of charts and questionnaire, in a booklet called "Passport."

\section{Evaluations}

Upon starting the program, patients underwent a standardized general neurology evaluation to determine and address potential medical causes for their memory loss and cognitive impairment such as sleep apnea, depression, and vitamin B12 deficiency. After this initial consultation, patients received a baseline battery of cognitive testing, MRI with quantitative volumetric analysis, and other tests, as medically indicated. For example, patients who were physically fit at baseline did not undergo cardiopulmonary exercise test, but those who had shortness of breath with exertion or had a sedentary lifestyle did. Those who had multiple vascular risk factors for stroke underwent carotid ultrasound. Those who had snoring, daytime hypersomnolence, insomnia, or other complaints about their sleeping underwent a formal polysomnogram. All patients had a quantitative EEG (Q-EEG), also known as brain mapping, at baseline. The deviations from normalized brain activity pattern were used to design the protocol for their neurofeedback therapy (6).

\section{Neuropsychological Testing}

Baseline neurocognitive battery of tests consisted of Trail-Making Test A and B (TMT-A and TMT-B), Hopkins Verbal Learning Test-Revised (HVLT-R), Brief Visuospatial Memory Test-Revised (BVMT-R), DelisKaplan Executive Function System (D-KEFS) tests of (i) Color-Word Interference Tests (Color Naming [COLOR], Word Reading [WORD], Inhibition) and (ii) Verbal Fluency Test (Letter Fluency [LETTER]). The same battery of tests was performed when patients completed the Brain Fitness Program.

\section{Brain MRI with NeuroQuant}

Patients who did not have a brain MRI within at least one year prior to the first evaluation underwent a brain MRI with NeuroQuant measurements of the hippocampus (7). A random subset of these patients $(n=17)$ underwent a post-program MRI with NeuroQuant to determine if there was a change in the size of their hippocampus.

\section{Three Main Sets of Interventions}

In this 12-week Brain Fitness Program, patients received two hours/week of cognitive skills training, one hour/week of counseling/brain coaching, and two hours/week of neurofeedback therapy (for a total of 60 hours over 12 weeks). Each patient's progress was discussed in weekly team conferences, especially at beginning, mid-point, and end of the program.

\section{Cognitive Skills Training}

Cognitive stimulation was performed using a standard computer-based cognitive training program called Captain's Log Professional System (www.braintrain. $\mathrm{com} /$ cognitive-training-research/). Widely used and validated in a number of previous studies, it consists of different types of game-like brain exercises to improve memory, attention, mental processing speed, and problem solving strategies (8). Each patient received a personalized protocol for a set of cognitive skills such as attention, working memory, or executive function. The treatment protocol was based on each patient's initial symptoms, their concerns, and their wish to improve in any specific cognitive domains (e.g., memory of names). This protocol was updated as the patient made improvements, and especially at patient's mid-program evaluation in six weeks.

\section{Brain Coaching/Counseling}

Brain coaching sessions were conducted by a master level psychometrist and consisted of meditation training, cognitive behavioral therapy, and education regarding a Mediterranean diet, exercise, stress reduction strategies, and improving sleep hygiene. Patients also received goaloriented training for particular circumstances and issues that were unique to their day-to-day life. To help increase their motivation, they were frequently reminded of their stated "purpose" for wanting to improve their cognitive performance (9).

\section{Neurofeedback}

Neurofeedback is a computer-based method for improving memory, attention, mood, and sleep through 
providing the patient with information about his/her EEG pattern, and then rewarding normalization of brain wave activity through visual and auditory feedback. Neurofeedback therapy has been shown to be helpful for elderly individuals with cognitive deficits (6). In this trial, neurofeedback sessions were conducted by trained EEG technicians, under the supervision of the neurologist and the co-director of the program (who had certification from Biofeedback Certification International Alliance, BCIA). Each patient received a personalized neurofeedback protocol based on their symptoms (e.g., anxiety, insomnia, or attention deficit) and baseline abnormalities in neuropsychological tests and Q-EEG. The baseline Q-EEG and neurofeedback interventions were applied with the latest version of the Brain Master hardware and software programs (www.BrainMaster. com).

\section{Statistical Analysis}

The performance scores on the neurocognitive tests were recorded as raw scores and then converted to standardized z-scores. Repeated measures nonparametric t-tests, with significance at 0.05 and Bonferroni correction, were performed for each component of the neurocognitive battery (ten tests) on the change in standardized z-score between the pre- and post-neurocognitive battery. Given the sample size of $120+$ participants, an increase of 1.65 standard deviations or more was necessary for statistical significance. The overall effectiveness for the Brain Fitness Program for each participant was the sum of the component tests with significant positive change. We also performed a Spearman correlational analysis of our data to determine if there were relationships between the changes in the volume of hippocampus and the changes in cognitive function.

\section{Results}

In this single-arm intervention trial, 127 elderly patients with an average age of $70.69(S D=10.53)$ and a diagnosis of MCI received cognitive training, neurofeedback, and brain coaching/counseling over a 12 -week period. Upon completing the program, patients on average improved in 4.68 out of the 10 cognitive domains. Patients with the highest baseline MMSE $(>28)$ and younger age $(<60)$ showed the most improvements. The post-program cognitive tests (Fig. 1.0) showed $84 \%$ of patients had statistically significant improvements in at least three of the 10 areas of cognitive function $(\mathrm{P}<0.05)$ (labeled as "high impact" group). Nine percent of patients had improvement in two areas of cognitive function (labeled as "moderate impact"). Seven percent of patients had statistically significant improvement in one or none of the areas of cognitive function (labeled as "low impact").

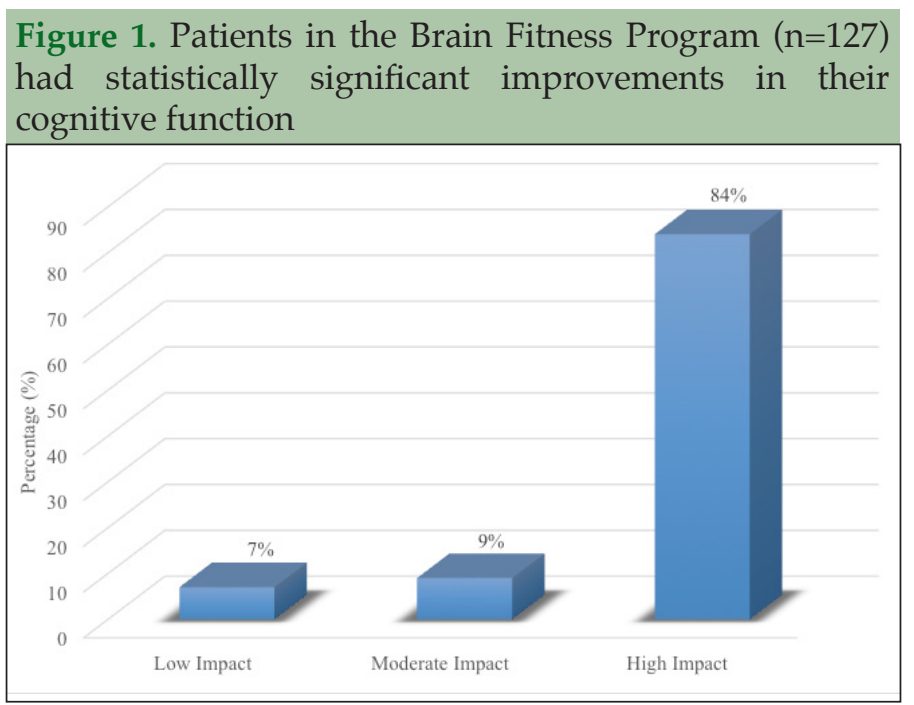

The battery of the neurocognitive evaluation used in this trial assessed 10 areas of cognitive function. In this trial, $84 \%$ of patients had improvements in three or more of the 10 areas of cognitive function (labeled as "high-impact"); $9 \%$ of patients had improvement in only two of the 10 areas of cognitive function (labeled as "moderate-impact"); and $7 \%$ of patients had improvement in only one or none of the 10 areas of cognitive function (labeled as "low-impact").

Figure 2. Before and after MRI results in one of the patients who completed the Brain Fitness Program

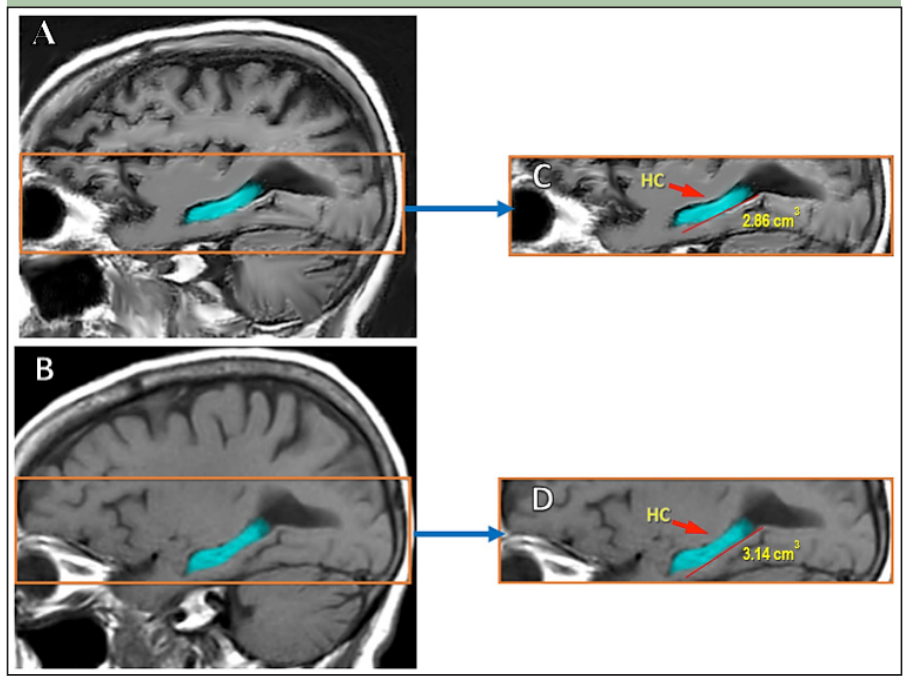

Post-program MRI with NeuroQuant showed an $8.6 \%$ growth in the volume of the hippocampus (HC) in one patient. (A): Baseline Brain MRI. (B): Post-program Brain MRI. (C): Higher magnification of baseline hippocampal volume. (D): Higher magnification of the post-program hippocampal volume

A random subset of 17 patients received a brain MRI after they completed the program. These post-program MRIs with NeuroQuant measurements showed that 9 of the 17 patients had an actual growth in the size of their hippocampus, with at least $1 \%$ expansion in its volume (data not shown). An example of a patient who had $8.6 \%$ growth in her hippocampus is shown in figure 2.0. Three of the 17 patients had no evidence of the expected hippocampal atrophy (no change) and five patients experienced the usual expected atrophy in the hippocampal volume.

A Spearman correlational analysis showed a correlation of 0.48 between changes in cognitive function 
and MRI, which suggests a trend but is not sufficient to meet the cutoff for statistical analysis $(\mathrm{p}=0.21)$.

\section{Discussion}

Results from the $127 \mathrm{MCI}$ patients who completed the 12-week Brain Fitness Program reveal that elderly patients with objective cognitive impairment have the capacity to improve their brain performance with an intensive set of treatments. In this population of elderly with $\mathrm{MCI}$, post-program cognitive tests showed that $84 \%$ of patients had statistically significant improvements in their attention, concentration, executive function, problem solving, or speed of cognitive processing. Postprogram MRI in a small subset of 17 patients showed that 12 patients experienced a reversal of hippocampal atrophy or an actual growth above its baseline volume.

The main strength of the Brain Fitness Program is its multi-faceted interventions. When the non-Alzheimer's pathologies such as sleep apnea, depression, diabetes, and concussion combine with varying degrees of Alzheimer's pathology and genetic factors, patients experience rapid cognitive decline and dementia $(1,2)$. It appears that when protective factors such as cognitive stimulation, stress reduction, improved diet, better fitness, and treatment of sleep issues are combined, patients do experience a reversal of their cognitive decline and can increase the volume of their hippocampus.

Another strength of the Brain Fitness Program is the personalized nature of its interventions. Patients are first asked to describe their symptoms, concerns, fears, and motivations for their desire to enhance their brain performance as well as the degree of their commitment for completing this intensive 3-month program. This information is then used in brain coaching/counseling sessions to establish a personalized protocol based on goal-oriented behavioral modifications $(9,10)$. Patients are encouraged to change their lifestyle habits on a gradual basis and receive weekly reminders about their "sense of purpose in life" in order to maintain motivation to adhere with the program.

The limitations of the current trial are its small size, lack of randomization, the absence of a control group, and the challenge to establish which of the interventions provided is most effective in treating patients with MCI. Patients had variable degrees of different comorbid medical conditions such as depression, sleep deficits, or cardiovascular disease. They also had variable degree of compliance with following instruction for treatment of their medical and neurological conditions. The level of family support to help them follow through with the program was also variable. However, given these limitations, the fact that a program in a real-life clinical setting can produce measurable results is quite encouraging. It is possible that the results may have been more significant if more patients were enrolled in this trial and they were randomized to either an active group or a control group. A large multicenter trial of the Brain Fitness Program may be able to answer which interventions would yield the most benefits for any individual patient in the shortest amount of time, and what dose of treatment would be sufficient to obtain clinically meaningful and long-lasting results.

An interesting observation in our trial is the fact that patients with a younger age and higher baseline MMSE seem to gain the most benefits with completing the Brain Fitness Program. These patients may have strong baseline brain reserve that can be rehabilitated and rebuilt $(1,2)$. Patients with advanced age and severe cognitive deficits may already have lost a significant portion of their brain reserve $(1,2)$. Such individuals may require increased frequency and magnitude of interventions for a much longer period of time, though it is not clear if they would ever rebound significantly.

To quantify and monitor change in hippocampal volume as a function of disease progression and biomarker of response to prevention and treatment trials, quantitative tools for hippocampal volume assessment are needed. Only two such programs are FDA cleared for use in patients: NeuroQuant and Neuroreader. In this paper, we used NeuroQuant which was the first FDA cleared software for this purpose. Neuroreader is a newer software program that has been validated against Delphi Consensus Criteria for manual segmentations of the hippocampus (11). Additionally, Neuroreader has an FDA cleared normative database that improves reliability of its Z-score calculations (12). Applying such tools in clinical practice for prevention purposes such as described in this paper is part of a larger goal of utilizing quantitative neuroimaging in brain aging and cognitive decline (13).

A unique feature of the Brain Fitness Program is its use of neurofeedback training for enhancing cognitive function and mood in elderly (6). In this intervention, patients undergo continuous EEG monitoring during a typical 40-minute session and receive auditory and visual feedback to move their brain wave activity toward the normal range. For example, a patient with stress, anxiety, or insomnia may have excessive levels of fast high-beta activity in his/her baseline Q-EEG. Through repeated neurofeedback sessions, with a protocol aimed to reward a reduction in high-beta activity, the patient gradually becomes calmer and more focused (14). Given the growing evidence for the long-lasting benefits of this non-pharmaceutical intervention (15), neurofeedback therapy has the potential to become an important tool for treatment of elderly with MCI.

In summary, these results for our Brain Fitness Program for patients with MCI further support the fact that a multi-disciplinary set of interventions has the potential to enhance cognitive function in elderly (4). It provides an additional incentive to initiate large placebocontrolled randomized clinical trials to evaluate the 
possibility that we can slow the rate of cognitive decline with aging, and grow the size of hippocampus, through an emphasis on diet, exercise, cognitive stimulation, neurofeedback, meditation, and counseling for stress reduction and having a purpose in life.

Acknowledgements: The authors would like to express their gratitude to the members of the Brain Fitness Program team which included Dr. Amy Cunningham, Rebekah Banerjee, Michelle Battaglia, Corissa Fanning, Dr. Mehdi Ghasemi, Laura Keene, Kristina Kosztyo, Crystal McDowell, Laurel Powers, Deepti Pradha, Dr. Margaret Timmons, Mark Trullinger, Lynnea Tull, Dr. Raya Wehbeh, and Dr. Zakiya Wynn. The authors would also like to thank Dr. Masoumeh Dejman and Ms. Christina Antoniades for their review of the manuscript, and especially to the patients who agreed to participate in this trial.

Funding: This trial was carried out in a clinical neurology practice in Maryland (USA), without any outside funding. No external support was used.

Conflicts of interest: Dr. Majid Fotuhi, Brooke Lubinski, and Tracy Riloff were employees of the clinical practice. Dr Raji is a consultant for Brainreader ApS. The authors otherwise have nothing to disclose regarding conflict of interests.

Ethical standards: The clinical trial described in this study was reviewed and approved by New England IRB (www.NEIRB.com).

\section{References}

1. Fotuhi, M., D. Do, and C. Jack, Modifiable factors that alter the size of the hippocampus with ageing. Nature Reviews Neurology, 2012. 8(4): p. 189-202.

2. Fotuhi, M., V. Hachinski, and P.J. Whitehouse, Changing perspectives regarding late-life dementia. Nature Reviews Neurology, 2009. 5(12): p. 649658.

3. Middleton, L.E. and K. Yaffe, Promising strategies for the prevention of dementia. Arch Neurol, 2009. 66(10): p. 1210-5.
4. Ngandu, T., et al., A 2 year multidomain intervention of diet, exercise, cognitive training, and vascular risk monitoring versus control to prevent cognitive decline in at-risk elderly people (FINGER): a randomised controlled trial. Lancet, 2015. 385(9984): p. 2255-63.

5. Albert, M.S., et al., The diagnosis of mild cognitive impairment due to Alzheimer's disease: recommendations from the National Institute on Aging-Alzheimer's Association workgroups on diagnostic guidelines for Alzheimer's disease. Alzheimers Dement, 2011. 7(3): p. 270-9.

6. Surmeli, T., et al., Quantitative EEG Neurometric Analysis-Guided Neurofeedback Treatment in Dementia: 20 Cases. How Neurometric Analysis Is Important for the Treatment of Dementia and as a Biomarker? Clin EEG Neurosci, 2015.

7. Ochs, A.L., et al., Comparison of Automated Brain Volume Measures obtained with NeuroQuant(R) and FreeSurfer. J Neuroimaging, 2015.

8. Lampit, A., C. Ebster, and M. Valenzuela, Multi-domain computerized cognitive training program improves performance of bookkeeping tasks: a matched-sampling active-controlled trial. Front Psychol, 2014. 5: p. 794.

9. Clare, L., et al., Goal-oriented cognitive rehabilitation in early-stage dementia: study protocol for a multi-centre single-blind randomised controlled trial (GREAT). Trials, 2013. 14: p. 152.

10. Clare, L., et al., The Agewell trial: a pilot randomised controlled trial of a behaviour change intervention to promote healthy ageing and reduce risk of dementia in later life. BMC Psychiatry, 2015. 15(1): p. 402.

11. Frisoni, G.B., et al., The EADC-ADNI Harmonized Protocol for manual hippocampal segmentation on magnetic resonance: evidence of validity. Alzheimers Dement, 2015. 11(2): p. 111-25.

12. Ahdidan, J., et al., Quantitative Neuroimaging Software for Clinical Assessment of Hippocampal Volumes on MR Imaging. J Alzheimers Dis, 2015.

13. Raji, C.A., et al., Hot Topics in Research: Preventive Neuroradiology in Brain Aging and Cognitive Decline. AJNR Am J Neuroradiol, 2015.

14. Cheon, E.J., B.H. Koo, and J.H. Choi, The Efficacy of Neurofeedback in Patients with Major Depressive Disorder: An Open Labeled Prospective Study. Appl Psychophysiol Biofeedback, 2015.

15. Steiner, N.J., et al., In-school neurofeedback training for ADHD: sustained improvements from a randomized control trial. Pediatrics, 2014. 133(3): p. 483-92. 